\title{
Une nouvelle ère pour le LHC et ses expériences
}

Alexandre Zabi ${ }^{(1)}$ (azabi@\|r.in2p3.fr) et Nicolas Morange ${ }^{(2)}$ (morange@lal.in2p3.fr)

(1) Membre de la collaboration CMS.

Laboratoire Leprince-Ringuet, UMR 7638, École polytechnique, 91128 Palaiseau Cedex

(2) Membre de la collaboration ATLAS.

Laboratoire de l'Accélérateur Linéaire, UMR 8607, Université Paris-Sud, BP 34, 91898 0rsay Cedex

Après deux ans d'arrêt

technique, le grand

collisionneur de hadrons

du CERN a repris ses opérations

en 2015, à une énergie et

une intensité bien plus élevées.

Les détecteurs géants ATLAS

et CMS ont été adaptés

à ce nouvel environnement,

par l'amélioration de leurs

systèmes de détection

et de leur capacité à sélectionner

en temps réel les collisions

les plus intéressantes.

L'analyse des données

collectées jusqu'à l'été 2016

a déjà fourni des résultats très attendus, sur la compréhension du boson de Higgs et sur

la recherche d'une nouvelle physique au-delà du Modèle Standard.
Une nouvelle ère commence pour le grand collisionneur de hadrons (LHC) du CERN. Après une première période d'exploitation en 2009-2012 (Run 1) réussie, puisque couronnée par la découverte du boson de Higgs, le LHC a redémarré en 2015 pour le Run 2 avec une énergie dans le centre de masse augmentée de $60 \%$, soit $13 \mathrm{TeV}$ : un record de plus pour l'accélérateur. Cette ouverture vers une nouvelle frontière en énergie représente un véritable défi pour les expériences, qui doivent faire face à des conditions extrêmes de fonctionnement. C'est aussi une fenêtre unique pour la compréhension du boson de Higgs et pour la recherche d'une nouvelle physique au-delà du Modèle Standard.

Un long arrêt technique de deux ans aura donc été nécessaire pour améliorer et consolider le LHC. L'augmentation de l'énergie des faisceaux a pour but d'élever les sections efficaces de production et d'étendre l'espace des phases disponible pour mettre en évidence de nouvelles particules. Un aspect particulièrement important pour mesurer avec précision des phénomènes rares, le taux de collisions produites par le LHC (ou luminosité), a lui aussi augmenté significativement. Un an après le redémarrage, la luminosité instantanée délivrée par la machine n'a cessé d'augmenter, jusqu'à une valeur record dépassant de $40 \%$ le fonctionnement nominal théorique du LHC!

\section{Des détecteurs toujours plus complexes}

Les détecteurs de particules des expériences ATLAS et CMS ont pour but de reconstruire le résultat des collisions. Afin d'être en mesure d'examiner minutieusement ces dernières, ces détecteurs géants identifient les particules au moyen de "trajectographes »

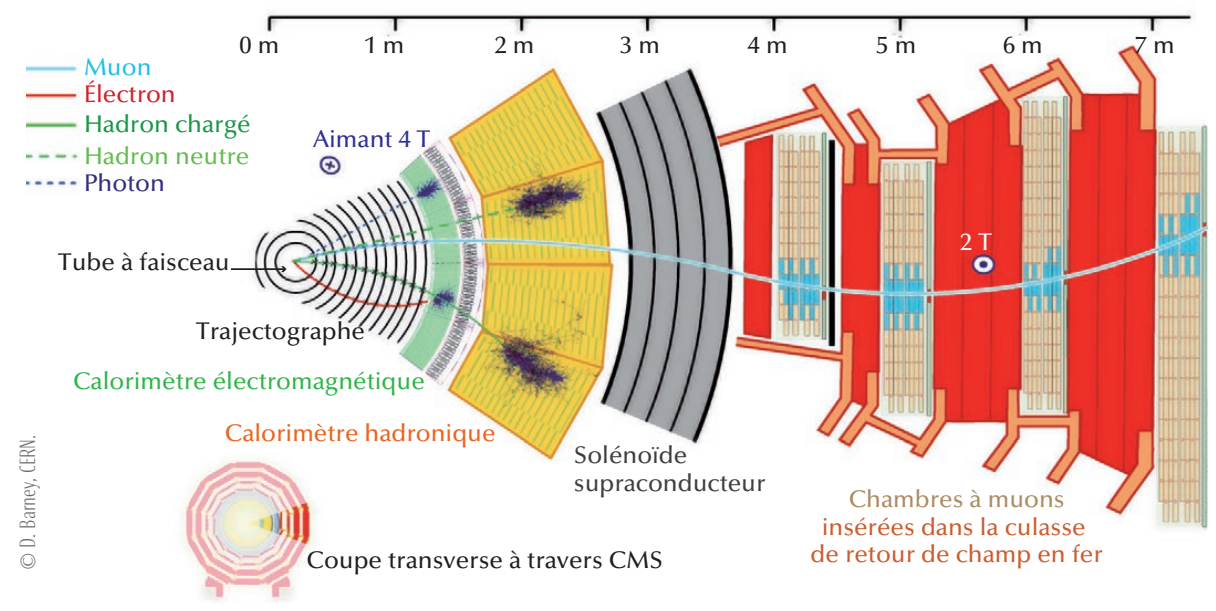

1. Représentation schématique d'une coupe du détecteur CMS. Du point de collision jusqu'à l'extérieur (de la gauche vers la droite sur la figure), les particules sont détectées par un trajectographe formé de plans de silicium, suivi par des calorimètres qui mesurent leurs énergies. La bobine supraconductrice permet de courber les trajectoires des particules et de mesurer leurs quantités de mouvement ainsi que leurs charges. Quatre couches de chambres à muons (de couleur beige sur la figure) sont placées autour de l'aimant afin de déterminer précisément leur impulsion. 


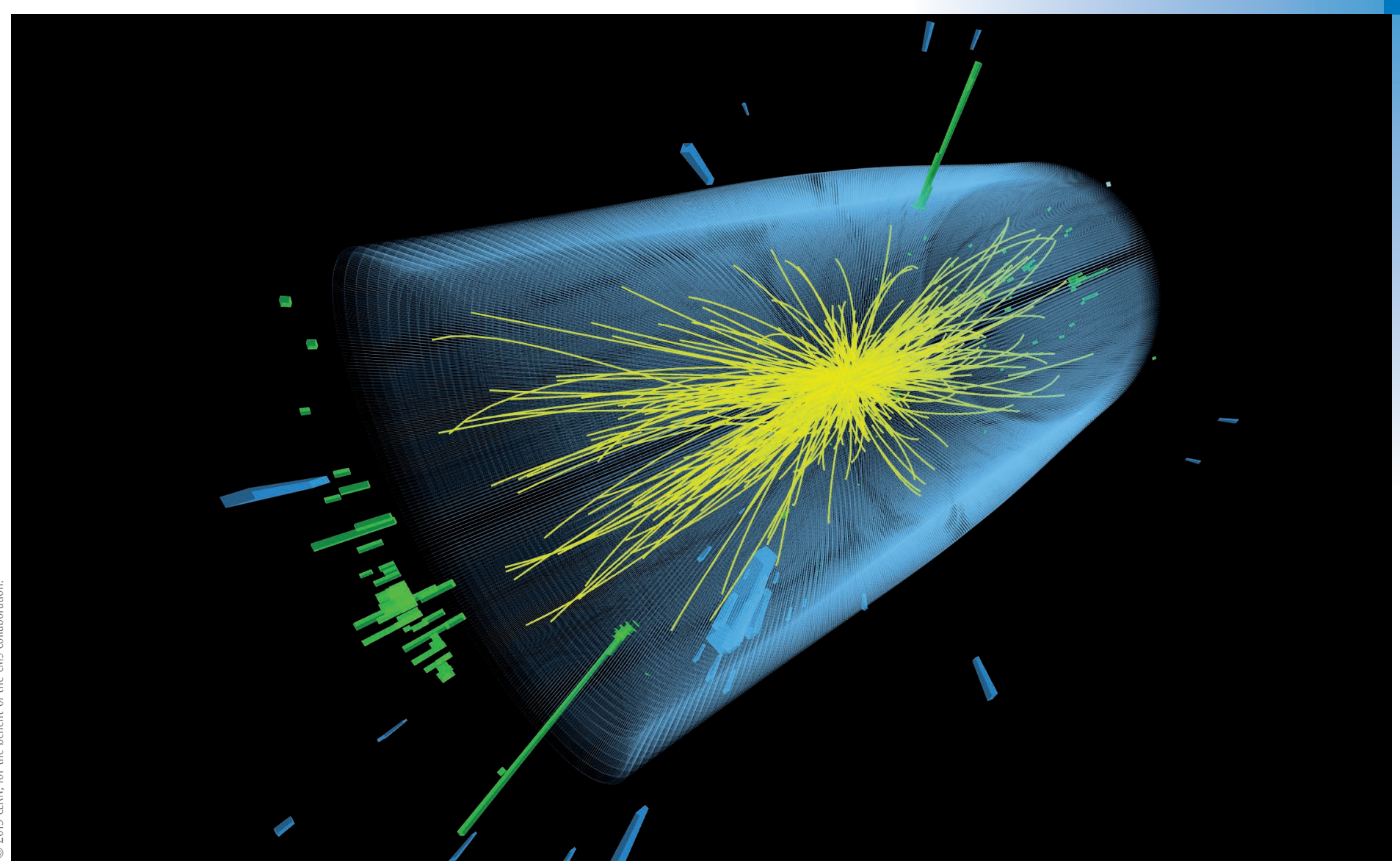

Représentation d'une collision avec un état final en deux photons de haute énergie (grandes barres vertes), d'une masse invariante proche de 750 GeV, enregistrée par le détecteur CMS en 2015. Les lignes jaunes représentent l'ensemble des traces de particules chargées reconstruites dans le détecteur. Les barres vertes et bleues représentent les dépôts d'énergie recueillis dans les calorimètres. La surface bleu clair représente le calorimètre électromagnétique de CMS, constitué de 76000 cristaux scintillants de PbW04.

et de calorimètres (fig. 1). La détection et la mesure simultanée des propriétés de centaines de particules représente une difficulté à surmonter pour les expériences. Or, dans l'idée d'atteindre des luminosités instantanées élevées, le nombre de protons par paquet est augmenté au niveau des injecteurs du LHC. Ceci a pour effet d'engendrer plusieurs collisions au moment où les paquets de protons se croisent. Généralement, une seule collision "dure ${ }^{(1)}$ " se produit et les collisions supplémentaires sont qualifiées d'" événements d'empilement ". Les particules produites par ces événements d'empilement ont une faible énergie et compliquent la tâche d'identification, au point d'affecter la résolution des mesures des détecteurs.

Dans le but de maintenir leurs performances de fonctionnement exceptionnelles établies lors du Run 1, les expériences ATLAS et CMS ont procédé à des modifications importantes de leurs détecteurs et des électroniques de lecture associées.

La reconstruction précise des traces des particules, ainsi que de leur point d'origine, est réalisée au moyen d'un "trajectographe " organisé en couches successives de modules de silicium. Au terme du Run 1, les modules de silicium placés au plus proche du tube à faisceau, donc sujets à une exposition permanente et intense aux neutrons provenant des collisions, ont été progressivement endommagés par les radiations. Afin de rétablir la capacité du détecteur à fournir un nombre de points de mesure suffisant le long de la trajectoire, les détecteurs de pixels ont été améliorés. La collaboration ATLAS a choisi d'insérer une couche supplémentaire de silicium très proche du tube à faisceau, alors que CMS a décidé de remplacer les modules défectueux. Dans les deux cas, ces opérations très délicates et particulièrement risquées, puisqu'il s'agit d'intervenir au cœur du détecteur, ont été accomplies à temps pour la reprise des collisions.

Un autre aspect de la prise de données concerne la sélection des collisions intéressantes au moyen d'un filtre communément appelé "système de déclenchement ". Un détecteur du calibre d'ATLAS ou de CMS est l'équivalent d'un appareil photo de 100 mégapixels opérant à la fréquence de $40 \mathrm{MHz}$. Le volume de données représente plusieurs téraoctets d'information par seconde, impossible à enregistrer dans son intégralité. Le système de déclenchement est utilisé pour analyser en direct les collisions en moins de $3 \mu$ s et choisir celles présentant un intérêt particulier. Ces systèmes de déclenchement sont composés de modules électroniques complexes associés à des ordinateurs puissants. Les avancées technologiques récentes ont permis aux expériences de remplacer une partie du système pour mettre en œuvre des algorithmes de déclenchement plus sophistiqués, capables d'examiner rapidement et en détail des événements remplis de plus de mille particules. Là encore, les équipes ont travaillé d'arrache-pied pour accomplir ces améliorations hautement techniques à temps pour les premières collisions.

Après deux ans d'arrêt, la reprise des collisions ne s'est pas faite sans heurts. Le détecteur CMS a, par exemple, souffert de défaillances du système de refroidissement de sa grande bobine supraconductrice, qui ont perturbé la prise de données en 2015. Mais ces problèmes font désormais partie du passé et, alors que la disponibilité des faisceaux du LHC en 2016 a dépassé toutes les prévisions (67\% contre 35\% en 2015), les détecteurs ATLAS et CMS ont pu enregistrer ces données de collisions avec plus de $90 \%$ d'efficacité. 


\section{\> \\ Des résultats très attendus sur les grandes questions du Modele Standard}

La découverte du boson de Higgs lors du Run 1 est un triomphe pour le Modèle Standard qui décrit les particules fondamentales et leurs interactions. Cependant ce modèle reste une théorie incomplète car, par exemple, il ne propose pas d'explication à la matière noire ni à la hiérarchie de masse entre les particules élémentaires. Un grand défi pour les physiciens des particules consiste donc à essayer de le mettre en défaut ! Pour cela, deux grandes possibilités : tester ses prédictions avec la plus grande précision possible et y chercher des déviations, ou bien tenter de mettre en évidence de nouvelles particules prédites par des théories de physique au-delà du Modèle Standard. Les données recueillies par les expériences en 2015 et 2016, exceptionnelles à la fois par l'énergie des collisions et par leur quantité, offrent des opportunités uniques dans ces deux voies.

La production d'un boson de Higgs est un événement rare dans les collisions. L'accumulation d'une gigantesque masse de données est donc une condition nécessaire pour mesurer précisément l'ensemble de ses propriétés, telles que ses taux de production et ses probabilités de désintégration dans différents états finals. Les résultats de l'analyse des données disponibles à l'été 2016, montrées par les collaborations lors de la grande conférence internationale ICHEP, atteignent déjà une précision similaire à celle du Run 1 pour les modes de désintégration les plus sensibles (désintégration en une paire de photons, ou en une paire d'électrons et une paire de muons) (fig. 2). Pour l'instant, les résultats des mesures sont parfaitement compatibles avec les prédictions du Modèle Standard. Mais trois fois plus de données sont maintenant disponibles, et leur analyse est attendue avec impatience. Par exemple, la sensibilité devrait être suffisante pour mettre en évidence deux propriétés très importantes du boson de Higgs : son canal de désintégration en paire de quarks $b$ (qui donne des indications sur sa largeur en énergie, inversement proportionnelle à son temps de vie), et sa production en association avec une paire de quarks top (qui fournit un test de compatibilité fondamental, tous modes de production confondus). Au-delà des études sur le boson de Higgs, la montée en énergie du LHC est un formidable levier pour les recherches

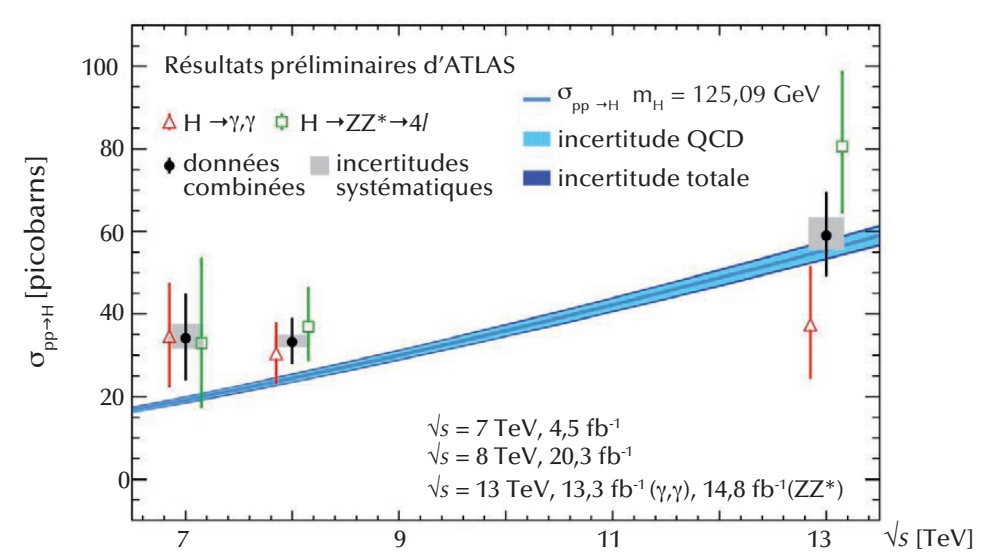

2. Mesure de la section efficace de production du boson de Higgs (H) aux différentes énergies explorées par le LHC $(\sqrt{ } s)$, dans ses canaux de désintégration en deux photons gammas (triangles rouges), en quatre leptons (carrés verts), et leur combinaison (cercles noirs). Les résultats du Run 2 (13 TeV) ont une précision relative déjà proche de celle obtenue au Run 1, et confirment les prédictions du Modèle Standard (courbe bleue).

de nouvelles particules, telles que celles prédites par les théories supersymétriques ou à dimensions supplémentaires. En effet, certaines de ces théories prédisent des particules massives, de quelques centaines de $\mathrm{GeV}$ à quelques $\mathrm{TeV}$, trop lourdes pour avoir été produites lors du Run 1. L'augmentation en énergie de l'accélérateur accroît considérablement l'espace des phases disponible, et le taux de production de ces particules massives pourrait alors devenir suffisant pour qu'elles soient détectées au-dessus des bruits de fond.

Une agitation s'est emparée de la communauté des physiciens quand l'analyse des données de 2015, montrée par les deux expériences ATLAS et CMS lors des conférences de l'hiver 2016, a révélé un excès d'événements à deux photons d'une masse invariante proche de $750 \mathrm{GeV}$. Était-ce le signe d'une nouvelle particule? Le fait qu'un excès similaire soit présent dans les deux expériences a échauffé les esprits, et l'information s'est propagée jusque dans les médias nationaux ${ }^{(2)}$. L'excès était bien visible sur les spectres, avec une signification statistique "locale " de 3,9 déviations standard (ou $\sigma$ ) pour ATLAS, et 3,4 $\sigma$ pour CMS. Cependant, comme l'analyse recherche de nouvelles particules sur une large gamme de masse, la probabilité d'observer quelque part dans le spectre un excès à cause d'une fluctuation statistique devient importante. De fait, les significations statistiques corrigées de ce "look-elsewhere effect" étaient beaucoup plus faibles (2,1 $\sigma$ pour ATLAS, 1,6 $\sigma$ pour CMS). L'analyse de quatre fois plus de données pour l'été 2016 a confirmé cette interprétation statistique, l'excès ayant disparu dans les deux expériences. Si pour l'instant aucune physique au-delà du Modèle Standard n'a été découverte, l'épisode de l'excès à $750 \mathrm{GeV}$ démontre le niveau de préparation des expériences, qui sont à l'affût de déviations dans de nombreux canaux très rapidement après la prise des données. Beaucoup d'analyses de recherche, notamment de supersymétrie, ont déjà une sensibilité largement supérieure à celle obtenue lors du Run 1.

En conclusion, les deux premières années du Run 2 ont été marquées par l'excellente performance du LHC pour délivrer des collisions à une énergie sans précédent, et la remarquable efficacité des détecteurs pour collecter des données de bonne qualité. Cela ouvre la voie à des mesures précises des propriétés du boson de Higgs, et à de nombreuses analyses de recherche d'une nouvelle physique, via la détection de nouvelles particules ou plus généralement de déviations par rapport aux prédictions du Modèle Standard. Les collaborations ATLAS et CMS ont montré qu'elles sont capables d'analyser rapidement de grandes quantités de données, et les prochaines séries de résultats, qui utiliseront l'ensemble des données de 2016, sont particulièrement attendues.

(1) Les protons étant des particules composites, seule une fraction de leur énergie est mise en jeu dans une collision. Les collisions (relativement rares) où cette énergie est une partie significative de celle des protons incidents, et qui produisent donc des particules de haute énergie dans l'état final, sont qualifiées de "dures".

(2) www.lemonde.fr/sciences/article/2016/03/21/ physique-effervescence-autour-d-une-particulemystere_4887196_1650684.html 